\title{
VS1-4
}

\section{Education on Holmium Laser Enucleation of the Prostate}

\author{
Department of Urology, Toho University school of medicine \\ Tadashi Ohira
}

Holmium laser enucleation of the prostate (HoLEP) is being performed more frequently as a transurethral treatment for benign prostatic hyperplasia. The surgical procedures can be divided into enucleation and morcellation, but unlike TUR-P, many studies have found that it takes 20 to 30 procedures to be proficient in HoLEP.

At present, most surgeons who perform HoLEP watch the surgery being performed at another hospital, and then learned the technique without the help of a mentor or instructor. A certain percentage of urology surgeons have given up performing HoLEP, and thus establishing educational and learning methods for HoLEP should further contribute to the use of HoLEP.

Because the endoscopic procedures for HoLEP are more complex than those for TUR-P, urology surgeons who are capable of excising $30-40 \mathrm{~g}$ of the gland in one hour are taught HoLEP at our hospital.

The important technical points for HoLEP include: (1) location of the incision on the urethral mucosa; (2) exposure of appropriate dissection line; and (3) maintenance of orientation. If a mucosal incision is placed at the wrong location, the incision cannot be repaired, and an appropriate dissection line cannot be exposed. At our hospital, an instructor places a mucosal incision to expose an appropriate dissection line, and then a learner performs the subsequent dissection procedures. While it is initially difficult to maintain the orientation of a large adenoma, lesions weighing about $100 \mathrm{~g}$ can be removed without any problem after sufficient practice.

Morcellation ensures a favorable field of view to avoid damaging the bladder, and this involves sufficient hemostasis following enucleation and filling the bladder with a perfusion solution. By focusing on these points, surgeons can learn HoLEP more rapidly than with previously reported methods.

\section{VS1-5}

\section{How to Learn Transurethral Detaching Prostatectomy (TUDP) in Saline}

\author{
Nippon Medical School, Tokyo \\ Yasunori Hiraoka \\ 1. Finding the landmark between the prostate lobes and the external sphincter muscle \\ 2. The partial detaching technique of adenoma with the prostate detaching blade from the surgical capsule and the \\ complete resection of the detached adenoma.
}

3. The complete detachment of adenoma (enucleation) and morcellation as well as HoLEP. 